\title{
A note on the risk management of CDOs
}

\author{
February 2007
}

\section{Jean-Paul Laurent}

ISFA Actuarial School, Université Lyon 1, 50, Avenue Tony Garnier, 69007 Lyon, France \& BNP Paribas, FIRST Credit, 10 Harewood Avenue, NW1 6AA London, England (e-mail: laurent.jeanpaul@free.fr)

\begin{abstract}
The purpose of this note is to describe a risk management procedure applicable to options on large credit portfolios such as CDO tranches on iTraxx or CDX. Credit spread risks are dynamically hedged using single name defaultable claims while default risk is kept under control thanks to diversification. The proposed risk management approach mixes ideas from finance and insurance and departs from standard approaches used in incomplete markets such as mean-variance hedging or expected utility maximisation. In order to ease the analysis and the exposure, default dates follow a multivariate Cox process.
\end{abstract}

Key words: CDOs, default risk, credit spread risk, dynamic hedging, incomplete markets, Cox process.

JEL Classification: G 13

\section{Introduction}

The hedging of defaultable claims is an involved topic (see Bielecki et al [2,3], BlanchetScalliet and Jeanblanc [7], Elouerkhaoui [10]), especially in a multivariate setting (see Bielecki et al [4]). To list a few issues at hand, we can mention the possibility of simultaneous defaults, contagion effects leading to jumps in credit spreads at default times, the occurrence of exogenous jumps in the default intensities and random recoveries.

While CDOs are a major development in financial markets, the hedging issues have not really been dealt with so far. The standard approach amongst credit derivatives trading desks involves portfolios based upon single name credit default swaps. The hedge ratios are

\footnotetext{
${ }^{1}$ The author gratefully thanks X. Burtschell, J-D. Fermanian, J. Gregory, S. Hitier, S. Hutt, M. Jeanblanc, J. Lebuchoux, M. Musiela, M. Rutkowski, A. Savine and the participants of the IAFE credit event in London and of Seminar Bachelier in Paris for helpful feedback and discussions. The usual disclaimer applies. This paper has an academic purpose and may not be related to the way BNP Paribas hedges its credit derivatives books.
} 
computed as sensitivities to marginal credit curves in a copula framework (Greenberg et al [14]). As a consequence, the main focus is put upon the credit spread hedging leaving aside default risks. This is paradoxical since in a copula model default intensities and thus credit spreads only depend upon past defaults (see Schönbucher and Schubert [23]). Using a martingale representation theorem with respect to the natural filtration of default times then leads to a hedging strategy concentrated upon the risk management of default risk (Bielecki et al [4]).

Given the large number of names in iTraxx on CDX indices, default of a single name has a small effect on the aggregate running loss. In other words, default risk is already partly diversified in large credit portfolios ${ }^{2}$. In this paper, default risk is managed thanks to portfolio diversification while credit spread risk is handled with dynamic hedging techniques. The core idea of the paper is to project the defaultable price processes onto the filtration generated by the default intensities and in a second step to hedge the smoothed payoff that only involves credit spread risks. We show that applying the latter dynamic hedging strategy to the actual defaultable price processes allows to control the hedging error (with respect to the number of names). When dealing with the risk management of CDOs, our approach reconciles market practice which focuses on credit spread risks and the theoretical emphasis upon default risk.

There is a large body of literature dedicated to large financial markets and completeness (see for instance Jarrow and Bättig [15]). Let us emphasize that while some of our results might be extended to an infinite number of names, this paper remains in a small market framework. Unlike Björk and Näslund [6] or De Donno [8] for example, hedging strategies are based on a finite and fixed number of assets and do not require well diversified portfolios which are not readily tradable in the market.

To keep things simple, default times follow a multivariate Cox process, thus leaving aside possible contagion effects. Thanks to the multivariate setting, we can cope with portfolio heterogeneity: credit deltas can actually depend upon the riskiness of the different names in the credit portfolio as was particularly apparent during the so-called correlation crisis in 2005 in the CDO market.

The paper is organized as follows. Sections 2 and 3 deal with defaultable price processes and their projections onto the filtration of credit spreads. The material there is rather standard and expository. Sect. 4 contains the main results related to the risk management of CDOs.

\footnotetext{
${ }^{2}$ The theory of infinitely granular credit portfolios is well-developed in the static case (see Gordy [13], Schönbucher [22], Vasicek [25]). Frey and Backaus [12], Jarrow, Lando and Yu [16] consider similar issues in a more dynamical setting.
} 


\section{Modelling of default times}

Let us consider a filtered probability space $\left(\Omega, \mathcal{A},\left(\mathcal{F}_{t}\right)_{0 \leq t \leq T}, P\right)$ with a fixed time horizon $T \in$ $\mathbb{R}^{+}$and some random variables $\tau_{1}, \ldots, \tau_{n}$ that denote the default times of $n$ obligors. For any $t \in[0, T], N_{i}(t)=1_{\left\{\tau_{i} \leq t\right\}}, i=1, \ldots, n$ denote the default indicators, $\mathcal{H}_{i, t}=\sigma\left(N_{i}(s), s \leq t\right)$, $\mathcal{H}_{t}=\mathcal{H}_{1, t} \vee \ldots \vee \mathcal{H}_{n, t}$ and $\mathcal{G}_{t}=\mathcal{F}_{t} \vee \mathcal{H}_{t}$. The background filtration $\mathcal{F}$ is typically associated with credit spread risks. The enlarged filtration $\mathcal{G}$ corresponds to the actual information of market participants.

We now assume the existence of an arbitrage-free financial market where a savings account and defaultable $T$ maturity zero-coupon bonds are being traded. A $T$ maturity defaultable bond on name $i$ is associated with a payment of $1_{\left\{\tau_{i}>T\right\}}$. For simplicity, we thereafter assume that the default-free interest rates are equal to zero. We denote by $l^{i}(t, T)$ the time $t$ price of an asset with a time $T$ payoff $N_{i}(T)=1_{\left\{\tau_{i} \leq T\right\}}{ }^{4}, i=1, \ldots, n$.

Assumption 1 There exists a probability $Q$ equivalent to $P$ such that:

1. for $i=1, \ldots, n$, the price processes of defaultable claims $l^{i}(., T)$ are $(Q, \mathcal{G})$ martingales:

$$
l^{i}(t, T)=E^{Q}\left[N_{i}(T) \mid \mathcal{G}_{t}\right]
$$

for $0 \leq t \leq T$.

2. the default times follow a multivariate Cox process:

$$
\tau_{i}=\inf \left\{t \in \mathbb{R}^{+}, U_{i} \geq \exp \left(-\int_{0}^{t} \lambda_{i, u} d u\right)\right\}, \quad i=1, \ldots, n
$$

where $\lambda_{1}, \ldots, \lambda_{n}$ are strictly positive, $\mathcal{F}$ - progressively measurable processes, $U_{1}, \ldots, U_{n}$ are independent random variables uniformly distributed on $[0,1]$ under $Q$ and $\mathcal{F}$ and $\sigma\left(U_{1}, \ldots, U_{n}\right)$ are independent under $Q$.

3. $E^{Q}\left[\left(\frac{d P}{d Q}\right)^{2}\right]<\infty^{5}$.

We will further denote by $\Lambda_{1}, \ldots, \Lambda_{n}$, the processes defined by:

$$
\Lambda_{i, t}=\int_{0}^{t} \lambda_{i, u} d u
$$

\footnotetext{
${ }^{3}$ For simplicity, the recovery rates are equal to zero.

${ }^{4}$ This payoff corresponds to a long position in a default-free $T$ maturity bond and a short position in a defaultable $T$ maturity bond.

${ }^{5}$ Let us remark that the equivalent martingale measure $Q$ is somehow independent of the number of names. This is related to the absence of asymptotic free lunch (see Klein [17]).
} 
for $t \geq 0, i=1, \ldots, n$.

The Cox process framework is now standard in finance (see Lando [20], the books by Bielecki and Rutkowski [5], Duffie and Singleton [9], Lando [19] and the references therein). More precisely, our setting corresponds to the conditionally independent default framework (see chapter 9 of Bielecki and Rutkowski [5]). As a consequence, $t \in \mathbb{R}^{+} \rightarrow \Lambda_{i, t \wedge \tau_{i}}$ is the $(Q, \mathcal{G})$ compensator of $\tau_{i}$, i.e. the processes $t \in \mathbb{R}^{+} \rightarrow N_{i}(t)-\Lambda_{i, t \wedge \tau_{i}}, i=1, \ldots, n$ are $(Q, \mathcal{G})$ martingales. $\lambda_{i}$ is the $(Q, \mathcal{G})$-intensity of the counting process $N_{i}$.

The multivariate Cox process framework is convenient since the $(H)$ hypothesis or martingale invariance property holds:

Lemma 2.1 Every $(Q, \mathcal{F})$ square integrable martingale is also a $(Q, \mathcal{G})$ square integrable martingale.

Proof See Appendix

Remark 2.1 Let us remark that there are no contagions effects under $Q$. The absence of contagion under $Q$ will further provide a split between default and credit spread risks for large portfolios.

Remark 2.2 While $\left(\tau_{1}, \ldots, \tau_{n}\right)$ is a multivariate Cox process under $Q$, it may not be a Cox process under $P$. For instance, we may have some contagion effects under $P$ (see Kusuoka $[18])$.

Remark 2.3 The joint survival function is such that $S\left(t_{1}, \ldots, t_{n}\right)=E^{Q}\left[\prod_{i=1}^{n} \exp \left(-\Lambda_{i, t_{i}}\right)\right]$ for $t_{1}, \ldots, t_{n} \in \mathbb{R}^{+}$. Since it is continuous, we must have $Q\left(\tau_{i}=\tau_{j}\right)=0$ for $i \neq j$ which precludes simultaneous defaults.

Remark 2.4 $E^{Q}\left[\left(\frac{d P}{d Q}\right)^{2}\right]<\infty$ states that the historical measure $P$ and the risk-neutral one $Q$ do not depart too much from one to another. On economic grounds, it constrains the magnitude of default risk premiums (see Jarrow, Lando and Yu [16] for a discussion).

We can now state the dynamics of the defaultable claims:

\section{Lemma 2.2 defaultable claim price dynamics}

$$
l^{i}(t, T)=\left(1-N_{i}(t)\right)\left(1-E^{Q}\left[\exp \left(\Lambda_{i, t}-\Lambda_{i, T}\right) \mid \mathcal{F}_{t}\right]\right)+N_{i}(t)
$$

for $0 \leq t \leq T$ and $i=1, \ldots, n$. 
Proof The $\sigma$ - fields $\mathcal{G}_{i, T}$ and $\mathcal{H}_{(-i), t}$ are conditionally independent given $\mathcal{G}_{i, t}$. Consequently, $Q\left(\tau_{i}>T \mid \mathcal{G}_{t}\right)=Q\left(\tau_{i}>T \mid \mathcal{G}_{i, t}\right)$. Since $\tau_{i}$ can be seen as a univariate Cox process, $Q\left(\tau_{i}>\right.$ $\left.T \mid \mathcal{G}_{i, t}\right)=1_{\left\{\tau_{i}>t\right\}} E^{Q}\left[\exp \left(\Lambda_{i, t}-\Lambda_{i, T}\right) \mid \mathcal{F}_{t}\right]$. We conclude by using $l^{i}(t, T)=E^{Q}\left[N_{i}(T) \mid\right.$ $\left.\mathcal{G}_{t}\right]=1-Q\left(\tau_{i}>T \mid \mathcal{G}_{t}\right)$.

Remark 2.5 From the monotonicity of conditional expectations, $0 \leq l^{i}(t, T) \leq 1$, for $i=1, \ldots, n$ and $0 \leq t \leq T$. Thus, $l^{i}(., T), i=1, \ldots, n$ are $(Q, \mathcal{G})$ square integrable martingales, with a jump at $\tau_{i}$.

Definition 2.1 predefault bond price dynamics We denote by:

$$
B_{i}(t, T)=E^{Q}\left[\exp \left(\Lambda_{i, t}-\Lambda_{i, T}\right) \mid \mathcal{F}_{t}\right]
$$

for $0 \leq t \leq t$

Remark 2.6 $B_{i}(t, T)$ is the defaultable bond price ${ }^{6}$ at time $t$ on the set $\left\{\tau_{i}>t\right\}$.

Hence, the dynamics of the defaultable claims simplifies to:

$$
l^{i}(t, T)=\left(1-N_{i}(t)\right)\left(1-B_{i}(t, T)\right)+N_{i}(t)
$$

\section{Portfolio dynamics}

Default-free processes. It will be convenient to consider the following default-free running loss processes:

Definition 3.1 We denote by $p^{i}($.$) , the default-free running loss process associated$ with name $i \in\{0, \ldots, n\}$, which is such that for $0 \leq t \leq T$ :

$$
p^{i}(t) \triangleq E^{Q}\left[N_{i}(t) \mid \mathcal{F}_{t}\right]=Q\left(\tau_{i} \leq t \mid \mathcal{F}_{t}\right)=1-\exp \left(-\Lambda_{i, t}\right) .
$$

Remark 3.1 The last equality is a direct consequence of Assumption (1). $p^{i}$ is a $\mathcal{F}$ adapted increasing process that, unlike $N_{i}$, does not jump at default times ${ }^{7}$.

We can also define the default free forward loss processes by:

\footnotetext{
${ }^{6}$ Associated with a payoff $1_{\left\{\tau_{i}>T\right\}}$ at time $T$

${ }^{7} p^{i}$ can be given a simple financial interpretation. Let us subdivide name $i$ into $K$ names each with nominal $1 / K$ and intensities equal to $\lambda_{i}$ but with independent thresholds $U_{i, k}$. We denote by $\tau_{i, k}$ the corresponding default dates. $\frac{1}{K} \sum_{k=1}^{K} 1_{\tau_{i, k} \leq t}$ converges a.s. to $p^{i}(t)$ as $K \rightarrow \infty$. In other words, $p^{i}(t)$ corresponds to some aggregate running loss where diversification holds at the level of name $i$.
} 
Definition 3.2 The default free $T$ forward loss process associated with name $i \in$ $\{0, \ldots, n\}$, denoted by $p^{i}(., T)$ is such that for $0 \leq t \leq T$ :

$$
p^{i}(t, T) \triangleq E^{Q}\left[p^{i}(T) \mid \mathcal{F}_{t}\right]=E^{Q}\left[N_{i}(T) \mid \mathcal{F}_{t}\right]=Q\left(\tau_{i} \leq T \mid \mathcal{F}_{t}\right)
$$

The second equality is a direct consequence of the definition of $p^{i}(T)$ and the law of total expectation.

Remark 3.2 From the monotonicity of conditional expectations, we readily see that $0 \leq$ $p^{i}(t, T) \leq 1$, for $i=1, \ldots, n$ and $0 \leq t \leq T$. Thus, the $p^{i}(., T)$ are $(Q, \mathcal{F})$ square integrable martingales and thus $(Q, \mathcal{G})$ square integrable martingales thanks to the martingale invariance property.

Remark 3.3 From the definition of $p^{i}(., T)$, we readily have:

$$
p^{i}(t, T)=E^{Q}\left[1-\exp \left(-\Lambda_{i, T}\right) \mid \mathcal{F}_{t}\right] .
$$

Lemma $3.1 p^{i}(t, T), i=1, \ldots, n$ are projections of the forward price processes $l^{i}(t, T)$ on $\mathcal{F}_{t}$ :

$$
p^{i}(t, T)=E^{Q}\left[l^{i}(t, T) \mid \mathcal{F}_{t}\right],
$$

for $i=1, \ldots, n$ and $0 \leq t \leq T$.

Proof Since $l^{i}(t, T)=E^{Q}\left[N_{i}(T) \mid \mathcal{G}_{t}\right]$, we only need to check that $p^{i}(t, T)=E^{Q}\left[N_{i}(T) \mid \mathcal{F}_{t}\right]$. We conclude from $\tau_{i} \leq T \Leftrightarrow U_{i} \geq \exp \left(-\Lambda_{i, T}\right)$ and the independence between $U_{i}$ and $\mathcal{F}_{t}$.

\section{Lemma 3.2}

$$
l^{i}(t, T)-p^{i}(t, T)=Z_{i}(t) B_{i}(t, T),
$$

for $i=1, \ldots, n$ and $0 \leq t \leq T$, where $Z_{i}(t)=\exp \left(-\int_{0}^{t} \lambda_{i, u} d u\right)-\left(1-N_{i}(t)\right)$, and $B_{i}(t, T)$ is the predefault bond price (see definition (2.1)).

Proof Since $p^{i}(t, T)=E^{Q}\left[N_{i}(T) \mid \mathcal{F}_{t}\right], l^{i}(t, T)-p^{i}(t, T)=E^{Q}\left[N_{i}(T) \mid \mathcal{G}_{t}\right]-E^{Q}\left[N_{i}(T) \mid \mathcal{F}_{t}\right]$, which yields:

$$
l^{i}(t, T)-p^{i}(t, T)=\left(\exp \left(-\int_{0}^{t} \lambda_{i, u} d u\right)-\left(1-N_{i}(t)\right)\right) \times E^{Q}\left[\exp \left(-\int_{t}^{T} \lambda_{i, u} d u\right) \mid \mathcal{F}_{t}\right] .
$$

Portfolio loss processes. Let us now consider portfolios based upon the previous individual processes. 
Definition 3.3 aggregate running loss process The aggregate running loss at time $t$ on a portfolio of $n$ names is such that for $0 \leq t \leq T$ :

$$
l_{n}(t) \triangleq \frac{1}{n} \sum_{i=1}^{n} N_{i}(t) .
$$

Remark 3.4 For simplicity, we have assumed that default exposures are equal to $\frac{1}{n}$ and that recovery rates are equal to zero. To emphasize the dependence upon the number of names $n$, we used the subscript in the running loss $l_{n}(t)$.

Definition 3.4 aggregate forward loss process The $T$ forward aggregate loss at time $t$ is such that for $0 \leq t \leq T$ :

$$
l_{n}(t, T) \triangleq E^{Q}\left[l_{n}(T) \mid \mathcal{G}_{t}\right]
$$

Remark 3.5 Since $0 \leq l_{n}(T) \leq 1$, we also have $0 \leq l_{n}(t, T) \leq 1$, for all $t \in[0, T]$ thanks to the monotonicity of conditional expectations. $l_{n}(., T)$ is thus a square integrable $(Q, \mathcal{G})$ martingale.

Remark 3.6 We readily have: $l_{n}(t, T)=\frac{1}{n} \sum_{i=1}^{n} l^{i}(t, T)$, which shows that $l_{n}(t, T)$ can be seen as a portfolio price process.

Definition 3.5 default-free aggregate running loss process The default free aggregate running loss at time $t$ is such that for $0 \leq t \leq T$ :

$$
p_{n}(t) \triangleq \frac{1}{n} \sum_{i=1}^{n} p^{i}(t) .
$$

Remark 3.7 $p_{n}(t)$ is a $\mathcal{F}$ - adapted increasing process. Unlike $l_{n}(t), p_{n}(t)$ does not jump at default times. $p_{n}(t)$ corresponds to the aggregate loss of a portfolio where the risk has been (infinitely) diversified at the name level.

\section{Option hedging}

We will further consider payoffs of the type $\left(l_{n}(T)-K\right)^{+}=\left(\frac{1}{n} \sum_{i=1}^{n} N_{i}(T)-K\right)^{+}$, for some $K \in[0,1]$ corresponding to so-called zero-coupon CDOs. Though zero-coupon CDOs are actually traded in the market, the most commonly traded CDOs involve more complex loss payments (see Laurent and Gregory [21]). To illustrate the risk management approach, we think it is more suitable to deal with simple payoffs.

Before proceeding further, let us state some technical lemmas: 


\section{Lemma 4.1}

$$
\left\|l_{n}(T)-p_{n}(T)\right\|_{2, Q}^{2}=\frac{1}{n^{2}} \sum_{i=1}^{n} E^{Q}\left[\left(1-\exp \left(-\Lambda_{i, T}\right)\right) \exp \left(-\Lambda_{i, T}\right)\right] .
$$

Proof See Appendix

Remark 4.1 Since $0 \leq\left(1-\exp \left(-\Lambda_{i, T}\right)\right) \exp \left(-\Lambda_{i, T}\right) \leq \frac{1}{2},\left\|l_{n}(T)-p_{n}(T)\right\|_{2, Q}^{2} \leq \frac{1}{2 n}$.

Remark 4.2 Lemma (4.1) simply states that the accumulated losses $l_{n}(T)$ can be well approximated by the $\mathcal{F}$ - adapted random variable $p_{n}(T)$. In other words, for large $n$, we can neglect default risks and concentrate on the credit spread risks embedded in $p_{n}(T)$.

From Lemma (4.1), we know that $p_{n}(T)$ is close to $l_{n}(T)$ for large $n$. We may thus consider the hedging of the payoff $\left(p_{n}(T)-K\right)^{+}$. Since $p_{n}(T)$ only involves credit spread risks and not default risks, it is more likely that we can hedge the latter payoff. More formally, we make the following assumption:

Assumption 2 There exists some bounded $\mathcal{F}$ - predictable processes $\theta_{1}(),. \ldots, \theta_{n}($.$) such$ that:

$$
\left(p_{n}(T)-K\right)^{+}=E^{Q}\left[\left(p_{n}(T)-K\right)^{+}\right]+\frac{1}{n} \sum_{i=1}^{n} \int_{0}^{T} \theta_{i}(t) d p^{i}(t, T)+z_{n}
$$

where $z_{n}$ is $\mathcal{F}_{T}$-measurable, of $Q$-mean zero and $Q$-strongly orthogonal to $p^{1}(., T), \ldots, p^{n}(., T)$.

Remark 4.3 The previous equation is simply the $(Q, \mathcal{F})$ Galtchouk - Kunita - Watanabe decomposition of $\left(p_{n}(T)-K\right)^{+} \cdot \theta_{1}(),. \ldots, \theta_{n}($.$) correspond to the optimal (Q, \mathcal{F})$ mean-variance hedging strategy based upon the abstract forward price processes $p^{1}(., T)$, $\ldots, p^{n}(., T)$.

Remark 4.4 If the default intensities $\lambda_{1}, \ldots, \lambda_{n}$ follow a multivariate Itô process, then the conditional on $\mathcal{F}$ default probabilities $p^{1}(., T), \ldots, p^{n}(. T)$ also follow a multivariate Itô process. Assuming that the diffusion matrix is of rank $n$, then $z_{n}=0$. This corresponds to some completeness of the credit spread market. In the case of jump-diffusion processes, the residual term $z_{n}$ usually differs from zero.

Remark 4.5 The key point in Assumption (2) is the boundedness of the $\theta_{i}$ 's. Let us remark that the individual credit deltas are equal to $\frac{\theta_{i}(t)}{n}$. For simplicity, we will thereafter assume that $0 \leq \theta_{i}() \leq$.1 for $i=1, \ldots, n$. This boundedness assumption is related to the propagation of convexity property. We refer to Bergenthum and Rüschendorf [1], Ekström and Tysk [11] and the references therein for some discussion in a multivariate jump diffusion setting. 
We now state another lemma related to the control of hedging errors:

Lemma 4.2 Under Assumptions (1) and (2), the following inequality holds:

$$
\left.\left\|\frac{1}{n} \sum_{i=1}^{n} \int_{0}^{T} \theta_{i}(t) d\left(l^{i}(t, T)-p^{i}(t, T)\right)\right\|_{2, Q}^{2} \leq \frac{1}{n^{2}} \sum_{i=1}^{n}\left(Q\left(\tau_{i} \leq T\right)+E^{Q}\left[B_{i}\right]_{T}\right]\right) .
$$

Proof See Appendix

We can now state our main result with respect to the hedging error:

Proposition 1 Under Assumptions (1) and (2), the hedging error $\varepsilon_{n}$ defined as:

$$
\varepsilon_{n}=\left(l_{n}(T)-K\right)^{+}-E^{Q}\left[\left(l_{n}(T)-K\right)^{+}\right]-\frac{1}{n} \sum_{i=1}^{n} \int_{0}^{T} \theta_{i}(t) d l^{i}(t, T),
$$

is such that $E^{P}\left[\left|\varepsilon_{n}\right|\right]$ is bounded by:

$$
\begin{aligned}
& \left.\frac{1}{\sqrt{2 n}}\left(1+\left(E^{Q}\left[\left(\frac{d P}{d Q}\right)^{2}\right]\right)^{1 / 2}\right)+\frac{1}{n}\left(E^{Q}\left[\left(\frac{d P}{d Q}\right)^{2}\right]\right)^{1 / 2}\left(\sum_{i=1}^{n}\left(Q\left(\tau_{i} \leq T\right)+E^{Q}\left[B_{i}\right]_{T}\right]\right)\right)^{1 / 2} \\
& +E^{P}\left[\left|z_{n}\right|\right] .
\end{aligned}
$$

Proof The proof is detailed in the Appendix. It is based on Lemmas (4.1) and (4.2) and on the following additional technical lemma:

Lemma 4.3 Let $A($.$) be a finite variation \mathcal{F}$ - adapted process such that $A(0)=0$ and $E^{Q}[A(T)]<\infty$. Let $\theta($.$) be a \mathcal{G}$ - adapted process such that $0 \leq \theta(t) \leq \bar{K}$, for $0 \leq t \leq T$ for some positive $\bar{K}$. Then, $E^{Q}\left[\int_{0}^{T} \theta(t) d A(t)\right]=E^{Q}\left[\int_{0}^{T} E^{Q}\left[\theta(t) \mid \mathcal{F}_{t}\right] d A(t)\right]$.

Proof See Appendix

Remark 4.6 The terms $E^{Q}\left[\left[B_{i}\right]_{T}\right]$ are related to the riskiness associated with credit spreads. The smaller the "volatility" associated with the credit spreads, the better the approximation hedge will be. Provided that the $E^{Q}\left[\left[B_{i}\right]_{T}\right]$ are uniformly bounded, that the risk premium term $E^{Q}\left[\left(\frac{d P}{d Q}\right)^{2}\right]$ also remains bounded and that the credit spread market is complete, the previous proposition states that the $\mathcal{L}^{1}(P)$ norm of the hedging error tends to zero at the speed $n^{-1 / 2}$ as $n$ tends to infinity. 
Remark 4.7 We apply the hedging strategy $\theta_{1}(),. \ldots, \theta_{n}($.$) to the actual defaultable claims$ with associated price processes $l^{1}(., T), \ldots, l^{n}(., T)$. When $\theta_{1}(t)=\ldots=\theta_{n}(t)=\theta(t)$, the aggregate portfolio becomes the single hedging instrument and $\frac{1}{n} \sum_{i=1}^{n} \int_{0}^{T} \theta_{i}(t) d l^{i}(t, T)=$ $\int_{0}^{T} \theta(t) d l_{n}(t, T)$. When $\theta(t)=1$, we simply hold the aggregate portfolio. However, even when $\tau_{1}, \ldots, \tau_{n}$ are exchangeable, there is no reason why the $\theta_{i}(t)$ should not depend upon the name $i$ for $t>0$. The name per name (or individual) model involves a larger number of credit deltas but can account for dispersion in the credit spreads which is problematic in a portfolio loss model. When the aggregate portfolio is actively traded (say in the case of iTraxx or CDX indices), one may use an exposure of $\theta(t)=\frac{1}{n} \sum_{i=1}^{n} \theta_{i}(t)$ to the index and of $\frac{\theta_{i}(t)-\theta(t)}{n}$ to the individual names in order to minimize transaction costs..

Projection of the option payoff on $\mathcal{F}_{T}$. We previously considered the risk management of the option payoff $\left(l_{n}(T)-K\right)^{+}$through the hedging of $\left(E^{Q}\left[l_{n}(T) \mid \mathcal{F}_{T}\right]-K\right)^{+}$. We might also have considered $E^{Q}\left[\left(l_{n}(T)-K\right)^{+} \mid \mathcal{F}_{T}\right]$. We show here that for large portfolios, these two approaches are equivalent.

\section{Lemma 4.4}

$$
E^{Q}\left[\left(p_{n}(T)-K\right)^{+} \mid \mathcal{F}_{t}\right] \leq E^{Q}\left[\left(l_{n}(T)-K\right)^{+} \mid \mathcal{F}_{t}\right]
$$

for all $t \in[0, T]$ and $K \in[0,1]$.

Proof Let us remark that $p_{n}(T)=E^{Q}\left[l_{n}(T) \mid \mathcal{F}_{T}\right]$. From conditional Jensen inequality, we have: $\left(p_{n}(T)-K\right)^{+} \leq E^{Q}\left[\left(l_{n}(T)-K\right)^{+} \mid \mathcal{F}_{T}\right]$ which yields the stated result.

Remark 4.8 $E^{Q}\left[\left(p_{n}(T)-K\right)^{+}\right] \leq E^{Q}\left[\left(l_{n}(T)-K\right)^{+}\right]$is consistent with the smaller "volatility" of $p_{n}(T)$ compared with $l_{n}(T)$.

\section{Lemma 4.5}

$$
\left\|E^{Q}\left[\left(p_{n}(T)-K\right)^{+} \mid \mathcal{F}_{t}\right]-E^{Q}\left[\left(l_{n}(T)-K\right)^{+} \mid \mathcal{F}_{t}\right]\right\|_{2, Q}^{2} \leq \frac{1}{2 n}
$$

for $0 \leq K \leq 1$ and $0 \leq t \leq T$.

Proof Let us denote by $u=\left|E^{Q}\left[\left(p_{n}(T)-K\right)^{+}-\left(l_{n}(T)-K\right)^{+} \mid \mathcal{F}_{t}\right]\right|$. Thanks to conditional Jensen inequality, $u \leq E^{Q}\left[\left|\left(p_{n}(T)-K\right)^{+}-\left(l_{n}(T)-K\right)^{+}\right| \mid \mathcal{F}_{t}\right]$. The latter quantity is smaller than $E^{Q}\left[\left|p_{n}(T)-l_{n}(T)\right| \mid \mathcal{F}_{t}\right]$ and thus than $\left(E^{Q}\left[\left(p_{n}(T)-l_{n}(T)\right)^{2} \mid \mathcal{F}_{t}\right]\right)^{1 / 2}$. Thanks to Lemma (4.1), we have $E^{Q}\left[\left(p_{n}(T)-l_{n}(T)\right)^{2} \mid \mathcal{F}_{T}\right]=\frac{1}{n^{2}} \sum_{i=1}^{n} \operatorname{Var}^{Q}\left[N_{i}(T) \mid \mathcal{F}_{T}\right] \leq$ $\frac{1}{2 n}$. Using the monotonicity of conditional expectations and the law of total expectation,

$$
E^{Q}\left[\left(p_{n}(T)-l_{n}(T)\right)^{2} \mid \mathcal{F}_{t}\right] \leq \frac{1}{2 n}
$$

and thus $u \leq \frac{1}{\sqrt{2 n}}$. As a consequence, $E^{Q}\left[u^{2}\right] \leq \frac{1}{2 n}$ which yields the stated result. 
Remark 4.9 We readily have some two-sided bounds:

$$
E^{Q}\left[\left(p_{n}(T)-K\right)^{+} \mid \mathcal{F}_{t}\right] \leq E^{Q}\left[\left(l_{n}(T)-K\right)^{+} \mid \mathcal{F}_{t}\right] \leq E^{Q}\left[\left(p_{n}(T)-K\right)^{+} \mid \mathcal{F}_{t}\right]+\frac{1}{\sqrt{2 n}}
$$

for all $t \in[0, T]$ and $K \in[0,1]$.

Remark 4.10 When the $\mathcal{F}$ - market is complete, $E^{Q}\left[\left(p_{n}(T)-K\right)^{+} \mid \mathcal{F}_{t}\right]$ is the time $t$ price of $\left(p_{n}(T)-K\right)^{+}$while $E^{Q}\left[\left(l_{n}(T)-K\right)^{+} \mid \mathcal{F}_{t}\right]$ is the time $t$ price of $E^{Q}\left[\left(l_{n}(T)-K\right)^{+} \mid \mathcal{F}_{T}\right]$. In such a complete credit spread market, the above price processes are unambiguously defined and are both $\mathcal{F}$ and $\mathcal{G}$ martingales thanks to the martingale invariance property. For large $n$, i.e. when the granularity of the portfolio is small, these two price processes are close (the inequalities hold almost surely).

Assumption 3 There exists some bounded $\mathcal{F}$-predictable processes $\tilde{\theta}_{1}(),. \ldots, \tilde{\theta}_{n}($.$) such that:$

$$
E^{Q}\left[\left(l_{n}(T)-K\right)^{+} \mid \mathcal{F}_{T}\right]=E^{Q}\left[\left(l_{n}(T)-K\right)^{+}\right]+\frac{1}{n} \sum_{i=1}^{n} \int_{0}^{T} \tilde{\theta}_{i}(t) d p^{i}(t, T)+\tilde{z}_{n}
$$

where $\tilde{z}_{n}$ is $\mathcal{F}_{T}$-measurable, of $Q$-mean zero and $Q$-strongly orthogonal to $p^{1}(., T), \ldots, p^{n}(., T)$.

Remark 4.11 The previous equation is the $(Q, \mathcal{F})$ - Galtchouk - Kunita - Watanabe decomposition of the payoff $E^{Q}\left[\left(l_{n}(T)-K\right)^{+} \mid \mathcal{F}_{T}\right]$. $\tilde{\theta}_{1}(),. \ldots, \tilde{\theta}_{n}($.$) correspond to the opti-$ mal $(Q, \mathcal{F})$ mean-variance hedging strategy based upon the abstract forward price processes $p^{1}(., T), \ldots, p^{n}(., T)$. When the $\mathcal{F}$ market is complete, $\tilde{z}_{n}=0$. As above, we will thereafter assume that $0 \leq \tilde{\theta}_{i}() \leq$.1 for $i=1, \ldots, n$.).

Proposition 2 Under Assumptions (1) and (3), the hedging error $\tilde{\varepsilon}_{n}$ defined as:

$$
\tilde{\varepsilon}_{n}=\left(l_{n}(T)-K\right)^{+}-E^{Q}\left[\left(l_{n}(T)-K\right)^{+}\right]-\frac{1}{n} \sum_{i=1}^{n} \int_{0}^{T} \tilde{\theta}_{i}(t) d l^{i}(t, T),
$$

is such that $E^{P}\left[\left|\tilde{\varepsilon}_{n}\right|\right]$ is bounded by:

$$
\left.\left(E^{Q}\left[\left(\frac{d P}{d Q}\right)^{2}\right]\right)^{1 / 2}\left(\sqrt{\frac{2}{n}}+\frac{1}{n}\left(\sum_{i=1}^{n}\left(Q\left(\tau_{i} \leq T\right)+E^{Q}\left[B_{i}\right]_{T}\right]\right)\right)^{1 / 2}\right)+E^{P}\left[\left|\tilde{z}_{n}\right|\right]
$$

Proof $\left|\left(l_{n}(T)-K\right)^{+}-E^{Q}\left[\left(l_{n}(T)-K\right)^{+} \mid \mathcal{F}_{T}\right]\right|$ can be bounded by:

$$
\left|\left(l_{n}(T)-K\right)^{+}-\left(p_{n}(T)-K\right)^{+}\right|+\left|\left(p_{n}(T)-K\right)^{+}-E^{Q}\left[\left(l_{n}(T)-K\right)^{+} \mid \mathcal{F}_{T}\right]\right| .
$$


Using Lemma (4.5) and the proof of Proposition (1), we have:

$$
E^{P}\left[\left|\left(l_{n}(T)-K\right)^{+}-E^{Q}\left[\left(l_{n}(T)-K\right)^{+} \mid \mathcal{F}_{T}\right]\right|\right] \leq\left(E^{Q}\left[\left(\frac{d P}{d Q}\right)^{2}\right]\right)^{1 / 2} \sqrt{\frac{2}{n}}
$$

The stochastic integral terms are treated as in Lemma (4.2).

Remark 4.12 This shows that when considering the risk-management of the CDO payoff $\left(l_{n}(T)-K\right)^{+}$we may as well choose the strategy $\theta_{1}(),. \ldots, \theta_{n}($.$) or the strategy \tilde{\theta}_{1}(),. \ldots, \tilde{\theta}_{n}($.$) .$

Study of $E^{Q}\left[\left(l_{n}(T)-K\right)^{+} \mid \mathcal{G}_{t}\right] . \quad E^{Q}\left[\left(l_{n}(T)-K\right)^{+} \mid \mathcal{G}_{t}\right]$ is the expectation of the payoff under "the" risk-neutral probability $Q^{8}$. Though there is no theoretical background based on dynamical replication at this stage, it is tempting to consider $E^{Q}\left[\left(l_{n}(T)-K\right)^{+} \mid \mathcal{G}_{t}\right]$ as the time $t$ "price" of the CDO tranche. We can actually relate $E^{Q}\left[\left(l_{n}(T)-K\right)^{+} \mid \mathcal{G}_{t}\right]$ and $E^{Q}\left[\left(l_{n}(T)-K\right)^{+} \mid \mathcal{F}_{t}\right]$. We cannot expect the same a.s. inequalities as above since on $\left\{l_{n}(t)=1\right\}, E^{Q}\left[\left(l_{n}(T)-K\right)^{+} \mid \mathcal{G}_{t}\right]$ reaches the upper bound $1-K$. We can however state that the processes are close with respect to the $\mathcal{L}^{2}(Q)$-norm.

\section{Proposition 3}

$$
\left\|E^{Q}\left[\left(l_{n}(T)-K\right)^{+} \mid \mathcal{G}_{t}\right]-E^{Q}\left[\left(l_{n}(T)-K\right)^{+} \mid \mathcal{F}_{t}\right]\right\|_{2, Q}^{2} \leq \frac{2}{n} .
$$

Proof Let us denote by $u=E^{Q}\left[\left(l_{n}(T)-K\right)^{+} \mid \mathcal{G}_{t}\right]-E^{Q}\left[\left(l_{n}(T)-K\right)^{+} \mid \mathcal{F}_{t}\right]$. From the martingale invariance property, $E^{Q}\left[\left(p_{n}(T)-K\right)^{+} \mid \mathcal{G}_{t}\right]=E^{Q}\left[\left(p_{n}(T)-K\right)^{+} \mid \mathcal{F}_{t}\right]$. Thus,

$|u| \leq\left|E^{Q}\left[\left(l_{n}(T)-K\right)^{+}-\left(p_{n}(T)-K\right)^{+} \mid \mathcal{G}_{t}\right]\right|+\left|E^{Q}\left[\left(p_{n}(T)-K\right)^{+}-\left(l_{n}(T)-K\right)^{+} \mid \mathcal{F}_{t}\right]\right|$.

From the proof of Lemma (4.5), we already have $\left|E^{Q}\left[\left(p_{n}(T)-K\right)^{+}-\left(l_{n}(T)-K\right)^{+} \mid \mathcal{F}_{t}\right]\right| \leq$ $\frac{1}{\sqrt{2 n}}$. Using conditional Jensen inequality yields:

$$
\left|E^{Q}\left[\left(l_{n}(T)-K\right)^{+}-\left(p_{n}(T)-K\right)^{+} \mid \mathcal{G}_{t}\right]\right| \leq E^{Q}\left[\left|\left(l_{n}(T)-K\right)^{+}-\left(p_{n}(T)-K\right)^{+}\right| \mid \mathcal{G}_{t}\right] .
$$

The right-hand side of the inequality is bounded by $E^{Q}\left[\left|l_{n}(T)-p_{n}(T)\right| \mid \mathcal{G}_{t}\right]$ which is itself bounded by $\left(E^{Q}\left[\left(l_{n}(T)-p_{n}(T)\right)^{2} \mid \mathcal{G}_{t}\right]\right)^{1 / 2}$. Thus $u^{2} \leq 2 E^{Q}\left[\left(l_{n}(T)-p_{n}(T)\right)^{2} \mid \mathcal{G}_{t}\right]+\frac{1}{n}$ and $E^{Q}\left[u^{2}\right] \leq \frac{2}{n}$ thanks to the law of total expectation and Lemma (4.1).

\footnotetext{
${ }^{8} Q$ is such that the traded defaultable bond price processes are $(Q, \mathcal{G})$-martingales.
} 


\section{Conclusion}

This note shows how the risk management of CDOs can be simplified when a large credit portfolio is involved. According to market practice, a greater consideration is given to the dynamic hedging of credit spread risks while default risks are mitigated. The Cox modelling assumption is crucial for disentangling default and credit spread risks. In this framework, defaults do not occur simultaneously and are not informative. There are no jumps in credit spreads and related contagion effects after default of one name.

Though this note suggests that for infinitely granular portfolios we can only care of credit spread risks, the practical application to CDX or iTraxx CDO tranches remains to be studied. As far as the number of names is concerned, we are an intermediate stance. It is likely that the credit spread risks should be managed by taking into account observed defaults. It is also likely that the hedging of a tranche should also take into account both default and credit spread risks, for instance using CDS of different maturities.

We did not specialize the credit spread dynamics nor detail the actual computation of the credit deltas. Using a Markovian framework would help understanding the various effects involved in the hedging of a tranche. Since we are likely to be in a high dimensional framework, efficient numerical approaches must be considered. This is left for future work.

\section{Appendix}

Proof of Lemma (2.1). An equivalent statement of the $(H)$ hypothesis is the following: for any $t \in \mathbb{R}^{+}$, for any $s \in[t, T]$ and any bounded $\mathcal{F}_{s}$ - measurable random variable $\xi$, we have: $E^{Q}\left[\xi \mid \mathcal{G}_{t}\right]=E^{Q}\left[\xi \mid \mathcal{F}_{t}\right]$. To show this, let us denote by $\mathcal{G}_{i, s}=\mathcal{F}_{s} \vee \mathcal{H}_{i, s}$ for some $i=1, \ldots, n$ (say $i=2$ ) and by $\mathcal{H}_{(-i), t}=\mathcal{H}_{1, t} \vee \ldots \mathcal{H}_{i-1, t} \vee \mathcal{H}_{i+1, t} \ldots \vee \mathcal{H}_{n, t}$. The $\sigma$ - fields $\mathcal{G}_{i, s}$ and $\mathcal{H}_{(-i), t}$ are conditionally independent given $\mathcal{G}_{i, t}$. Consequently, since $\xi$ is $\mathcal{G}_{i, s}$ - measurable, we have $E^{Q}\left[\xi \mid \mathcal{G}_{t}\right]=E^{Q}\left[\xi \mid \mathcal{G}_{i, t} \vee \mathcal{H}_{(-i), t}\right]=E^{Q}\left[\xi \mid \mathcal{G}_{i, t}\right]$. Now, we are back in a univariate Cox process framework and it is well known that $E^{Q}\left[\xi \mid \mathcal{G}_{i, t}\right]=E^{Q}\left[\xi \mid \mathcal{F}_{t}\right]$.

Proof of Lemma (4.1). Since the means of $l_{n}(T)$ and $p_{n}(T)$ are equal, we need to consider $\operatorname{Var}^{Q}\left[l_{n}(T)-p_{n}(T)\right]$ which, thanks to the law of total variance, is equal to:

$$
\operatorname{Var}^{Q}\left[E^{Q}\left[l_{n}(T)-p_{n}(T) \mid \mathcal{F}_{T}\right]\right]+E^{Q}\left[\operatorname{Var}^{Q}\left[l_{n}(T)-p_{n}(T) \mid \mathcal{F}_{T}\right]\right] .
$$

The first term is equal to zero and:

$$
\operatorname{Var}^{Q}\left[l_{n}(T)-p_{n}(T) \mid \mathcal{F}_{T}\right]=\operatorname{Var}^{Q}\left[l_{n}(T) \mid \mathcal{F}_{T}\right]=\frac{1}{n^{2}} \sum_{i=1}^{n} \operatorname{Var}^{Q}\left[N_{i}(T) \mid \mathcal{F}_{T}\right]
$$


from the conditional independence of the $N_{i}(T)$ given $\mathcal{F}_{T}$. We conclude using:

$$
\operatorname{Var}^{Q}\left[N_{i}(T) \mid \mathcal{F}_{T}\right]=\left(1-\exp \left(-\Lambda_{i, T}\right)\right) \exp \left(-\Lambda_{i, T}\right)
$$

Proof of Lemma (4.3). Let us consider some partition $0<\ldots t_{j-1}<t_{j}<\ldots<T$ of $[0, T]$. Using linearity of expectations and the law of total expectation,

$$
E^{Q}\left[\sum_{j} \theta\left(t_{j-1}\right)\left(A\left(t_{j}\right)-A\left(t_{j-1}\right)\right)\right]=E^{Q}\left[\sum_{j} E^{Q}\left[\theta\left(t_{j-1}\right) \mid \mathcal{F}_{t_{j-1}}\right]\left(A\left(t_{j}\right)-A\left(t_{j-1}\right)\right)\right] .
$$

As the mesh of the partition tends to zero, the two discrete sums converge (for any state of the world) to the Stieltjes integrals $\int_{0}^{T} \theta(t) d A(t)$ and $\int_{0}^{T} E^{Q}\left[\theta(t) \mid \mathcal{F}_{t}\right] d A(t)$. When $A$ is increasing, we conclude using Lebesgue theorem. This extends to the finite variation case by linearity.

Proof of Lemma (4.2). From Kunita and Watanabe, $\left\|\sum_{i=1}^{n} \int_{0}^{T} \theta_{i}(t) d\left(l^{i}(t, T)-p^{i}(t, T)\right)\right\|_{2, Q}^{2}$ can be written as:

$$
\sum_{i, j=1}^{n} E^{Q}\left[\int_{0}^{T} \theta_{i}(t) \theta_{j}(t) d\left[l^{i}(t, T)-p^{i}(t, T), l^{j}(t, T)-p^{j}(t, T)\right]_{t}\right]
$$

which involves the quadratic covariations of the $(Q, \mathcal{G})$ square integrable bounded martingales $l^{i}(., T)-p^{i}(., T)$. Since $l^{i}(t, T)-p^{i}(t, T)=Z_{i}(t) B_{i}(t, T)$ (see Lemma (3.2)), when $i \neq j$, the quadratic covariation $\left[l^{i}(., T)-p^{i}(., T), l^{j}(., T)-p^{j}(., T)\right]$ involves only the quadratic covariation of $B_{i}(t, T)$ and $B_{j}(t, T)^{9}$ :

$$
\left[l^{i}(., T)-p^{i}(., T), l^{j}(., T)-p^{j}(., T)\right]_{t}=Z_{i}(t) Z_{j}(t)\left[B_{i}, B_{j}\right]_{t}, i \neq j .
$$

The quadratic variation of $l^{i}(t, T)-p^{i}(t, T)$ involves two terms, one associated with the quadratic variation of $Z_{i}(t)$ (or default risk) and one associated with the quadratic variation of $B_{i}(t, T)$ or credit spread risk. The quadratic variation associated with the default indicator part of $l^{i}(t, T)-p^{i}(t, T), Z_{i}(t)$, is equal to $N_{i}(t)$.

We can write:

$$
\sum_{i, j=1}^{n} \theta_{i}(t) \theta_{j}(t) d\left[l^{i}(., T)-p^{i}(., T), l^{j}(., T)-p^{j}(., T)\right]_{t}=\sum_{i, j=1}^{n} A_{i, j} d\left[B_{i}, B_{j}\right]_{t}+\sum_{i=1}^{n} D_{i} d N_{i}(t),
$$

\footnotetext{
${ }^{9}$ This is the core of the Cox modelling: there are no simultaneous defaults; defaults are not contagious. This allows for diversification of default risk in large portfolios. This holds even if the predefault bond prices have common jump components.
} 
where the $A_{i, j}, D_{i}$ are given by: $A_{i, j}=\theta_{i}(t) \theta_{j}(t) Z_{i}(t) Z_{j}(t)$ and $D_{i}=\theta_{i}^{2}(t) B_{i}^{2}(t, T)$.

We can thus write:

$$
\left\|\sum_{i=1}^{n} \int_{0}^{T} \theta_{i}(t) d\left(l^{i}(t, T)-p^{i}(t, T)\right)\right\|_{2, Q}^{2}=E^{Q}\left[\int_{0}^{T}\left(\sum_{i, j=1}^{n} A_{i, j} d\left[B_{i}, B_{j}\right]_{t}+\sum_{i=1}^{n} D_{i} d N_{i}(t)\right)\right] .
$$

Let us firstly consider the terms $E^{Q}\left[\int_{0}^{T} A_{i, j} d\left[B_{i}, B_{j}\right]_{t}\right]$. Thanks to Lemma (4.3),

$$
E^{Q}\left[\int_{0}^{T} A_{i, j} d\left[B_{i}, B_{j}\right]_{t}\right]=E^{Q}\left[\int_{0}^{T} E^{Q}\left[A_{i, j} \mid \mathcal{F}_{t}\right] d\left[B_{i}, B_{j}\right]_{t}\right] .
$$

Since $\theta_{i}$ and $\theta_{j}$ are $\mathcal{F}$-adapted, $E^{Q}\left[A_{i, j} \mid \mathcal{F}_{t}\right]=\theta_{i}(t) \theta_{j}(t) E^{Q}\left[Z_{i}(t) Z_{j}(t) \mid \mathcal{F}_{t}\right]$. Thanks to the conditional independence of default times upon $\mathcal{F}$,

$$
E^{Q}\left[Z_{i}(t) Z_{j}(t) \mid \mathcal{F}_{t}\right]=\operatorname{Cov}^{Q}\left(N_{i}(t), N_{j}(t) \mid \mathcal{F}_{t}\right)=0
$$

for $i \neq j$ and $0 \leq t \leq T$. As a consequence, $E^{Q}\left[A_{i, j} \mid \mathcal{F}_{t}\right]=0$ for $i \neq j$ and:

$$
E^{Q}\left[\int_{0}^{T} \sum_{i, j=1}^{n} A_{i, j} d\left[B_{i}, B_{j}\right]_{t}\right]=E^{Q}\left[\int_{0}^{T} \sum_{i=1}^{n} \theta_{i}^{2}(t) Z_{i}^{2}(t) d\left[B_{i}\right]_{t}\right] .
$$

Since $0 \leq \theta_{i}^{2}(t) Z_{i}^{2}(t) \leq 1, E^{Q}\left[\int_{0}^{T} \sum_{i=1}^{n} \theta_{i}^{2}(t) Z_{i}^{2}(t) d\left[B_{i}\right]_{t}\right] \leq \sum_{i=1}^{n}\left[B_{i}\right]_{T}$. Similarly, since $0 \leq D_{i} \leq 1, E^{Q}\left[\int_{0}^{T}\left(\sum_{i=1}^{n} D_{i} d N_{i}(t)\right)\right] \leq \sum_{i=1}^{n} Q\left(\tau_{i} \leq T\right)$. The proof is complete.

Proof of Proposition (1). Using triangle inequalities, we readily bound $E^{P}\left[\left|\varepsilon_{n}\right|\right]$ by:

$$
\begin{aligned}
& E^{P}\left[\left|l_{n}(T)-p_{n}(T)\right|\right]+E^{Q}\left[\left|l_{n}(T)-p_{n}(T)\right|\right]+\frac{1}{n} E^{P}\left[\left|\sum_{i=1}^{n} \int_{0}^{T} \theta_{i}(t) d\left(l^{i}(t, T)-p^{i}(t, T)\right)\right|\right] \\
& +E^{P}\left[\left|z_{n}\right|\right] .
\end{aligned}
$$

As for the term $E^{P}\left[\left|l_{n}(T)-p_{n}(T)\right|\right]$, we have:

$$
E^{P}\left[\left|l_{n}(T)-p_{n}(T)\right|\right] \leq\left(E^{Q}\left[\left(\frac{d P}{d Q}\right)^{2}\right]\right)^{1 / 2} \times\left\|l_{n}(T)-p_{n}(T)\right\|_{2, Q} .
$$

Using Lemma (4.1), we can thus bound $E^{P}\left[\left|l_{n}(T)-p_{n}(T)\right|\right]+E^{Q}\left[\left|l_{n}(T)-p_{n}(T)\right|\right]$ by:

$$
\frac{1}{\sqrt{2 n}}\left(1+\left(E^{Q}\left[\left(\frac{d P}{d Q}\right)^{2}\right]\right)^{1 / 2}\right)
$$


Let us now concentrate upon the dynamic hedging strategy term. From Cauchy-Schwarz inequality, we get:

$$
\begin{aligned}
& E^{P}\left[\mid \sum_{i=1}^{n} \int_{0}^{T} \theta_{i}(t) d\left(l^{i}(t, T)-p^{i}(t, T) \mid\right]\right. \\
& \leq\left(E^{Q}\left[\left(\frac{d P}{d Q}\right)^{2}\right]\right)^{1 / 2} \times\left\|\sum_{i=1}^{n} \int_{0}^{T} \theta_{i}(t) d\left(l^{i}(t, T)-p^{i}(t, T)\right)\right\|_{2, Q}
\end{aligned}
$$

Lemma (4.2) allows to conclude.

\section{References}

1. Bergenthum, J., Rüschendorf, L.: Comparison of option prices in semimartingale models, Finance Stochast., 10, 222-249 (2006)

2. Bielecki, T. R., Jeanblanc, M., Rutkowski, M.: Hedging of defaultable claims, ParisPrinceton lectures on mathematical finance 2003, Lecture Notes in Mathematics 1847, Springer 2004, pp. 1-132

3. Bielecki, T. R., Jeanblanc, M., Rutkowski, M.: Hedging of credit derivatives in models with totally unexpected defaults, In Stochastic Processes and Applications to Mathematical Finance, J. Akahori et al., eds, World Scientific 2006, pp. 35-100

4. Bielecki, T. R., Jeanblanc, M., Rutkowski, M.: Hedging of basket credit derivatives in credit default swap markets, working paper, Evry University (2006)

5. Bielecki, T. R., Rutkowski, M.: Credit risk: modeling, valuation and hedging, Springer 2002

6. Björk, T., Näslund, B.: Diversified portfolios in continuous time, European Finance Review, 1, 361-387 (1998)

7. Blanchet-Scalliet, C., Jeanblanc,M.: Hazard rate for credit risk and hedging defaultable contingent claims, Finance Stochast., 8, 145-159 (2004)

8. De Donno, M.: A note on completeness in large financial markets, Math. Finance, 14, 295-315 (2004)

9. Duffie, D., Singleton, K.J.: Credit risk: pricing, measurement and management, Princeton University Press 2003 
10. Elouerkhaoui, Y.: Étude des problèmes de corrélation et d'incomplétude dans les marchés de crédit, PhD thesis, University Paris Dauphine (2006)

11. Ekström, E., Tysk, J.: Convexity preserving jump diffusion models for option pricing, arXiv e-print (2006)

12. Frey, R., Backhaus, J.: Portfolio credit risk models with interacting default intensities: a Markovian approach, working paper, University of Leipzig (2004)

13. Gordy, M.: A risk-factor model foundation for ratings-based bank capital rules, J. Financial Intermediation, 12, 199-232 (2003)

14. Greenberg, A., O'Kane, D., Schloegl, L.: LH+: a fast analytical model for CDO hedging and risk management, Lehman Brothers Quantitative Research Quarterly (2004)

15. Jarrow, R.A., Bättig, R.J.: The second fundamental theorem of asset pricing: a new approach, Rev. Financial Stud., 12, 1219-1235 (1999)

16. Jarrow, R.A., Lando, D., Yu, F.: Default risk and diversification: theory and applications, Math. Finance, 15, 1-26 (2005)

17. Klein, I.: A fundamental theorem of asset pricing for large financial markets, Math. Finance, 10, 443-458 (2000)

18. Kusuoka, S.: A remark on default risk models, Advances in mathematical economics, Vol. 1, 69-82 (1999)

19. Lando, D.: Credit risk modeling, Princeton University Press 2004

20. Lando, D.: Three essays on contingent claims pricing, PhD Thesis, Cornell University (1994)

21. Laurent, J-P., Gregory, J.,: Basket default swaps, CDOs and factor copulas, J. Risk, $7(4), 103-122(2005)$

22. Schönbucher, P.: Taken to the limit: simple and not-so simple loan loss distributions, working paper, University of Bonn (2002)

23. Schönbucher, P., Schubert, D.: Copula-dependent default risk in intensity models, working paper, University of Bonn (2001)

24. Vasicek, O.: Probability of loss on loan portfolio, working paper, Moody's KMV (1987) 